\title{
67. \\ NOTE SUR LES FONCTIONS ELLIPTIQUES.
}

[From the Journal für die reine und angewandte Mathematik (Crelle), tom. xxxvII. (1848), pp. 58-60.]

SoIT $x=\sqrt{ } k \sin$ am $u$ et $\alpha=k+\frac{1}{k}$ : la fonction $\sqrt{ } k \sin$ am $n u$ (où $n$ est un entier), peut être exprimée sous forme d'une fraction dont le dénominateur est une fonction rationnelle et entière par rapport à $x$ et $\alpha$. En exprimant ce dénominateur par $z$, on aura

$$
n^{2}\left(n^{2}-1\right) x^{2} z+\left(n^{2}-1\right)\left(\alpha x-2 x^{3}\right) \frac{d z}{d x}+\left(1-\alpha x^{2}+x^{4}\right) \frac{d^{2} z}{d x^{2}}-2 n^{2}\left(\alpha^{2}-4\right) \frac{d z}{d \alpha}=0 \ldots
$$

Cette équation est due à M. Jacobi, (voyez les deux mémoires "Suite des notices sur les fonctions elliptiques," t. III. [1828] p. 306 et t. IV. [1829] p. 185.)

En essayant d'intégrer cette équation à moyen d'une suite ordonnée suivant les puissances de $x$, et en considérant en particulier les cas $n=2,3,4$ et 5 , j'ai trouvé que les différentes puissances de $\alpha$ se présentent et disparaissent d'une manière assez bizarre: (voyez mon mémoire "On the theory of elliptic functions," Cambridge and Dublin Math. Journal, t. II. [1847], p. 256, [45].) J'ai reconnu depuis que cela vient de ce que la valeur de $z$ est composée de plusieurs séries indépendantes; une quelconque de ces séries ordonnée selon les puissances descendantes de $\alpha$ va à l'infini; mais, en combinant les différentes séries, les termes qui contiennent les puissances négatives de $\alpha$ se détruisent. Par rapport à $x$ chacune de ces séries ne contient que des puissances paires et positives, car les puissances négatives qui y entrent apparemment, se réduisent toujours à zéro. En effet, on satisfait à l'équation (1) en supposant pour $z$ une expression de la forme

$$
z_{s}=2^{2 s(n-2 s)} \alpha^{s(n-2 s)} Z_{0} \ldots+(-1)^{q} 2^{2 s(n-2 s)-2 q} \alpha^{s(n-2 s)-q} \cdot Z_{q} \ldots
$$


(où $s$ est arbitraire). Cela donne pour $Z_{q}$ l'équation aux différences mêlées:

$$
\begin{aligned}
& {\left[x^{2} \frac{d^{2}}{d x^{2}}-\left(n^{2}-1\right) x \frac{d}{d x}+2 n^{2} s(n-2 s)-2 n^{2} q\right] Z_{q} } \\
+ & {\left[\left\{n^{2}\left(n^{2}-1\right) x^{2}+x^{4} \frac{d^{2}}{d x^{2}}-\left(2 n^{2}-2\right) x^{3} \frac{d}{d x}\right\}+\frac{d^{2}}{d x^{2}}\right] 4 Z_{q-1} } \\
- & 128 n^{2}[s(n-2 s)-q+2] Z_{q-2}=0 .
\end{aligned}
$$

Pour intégrer cette équation, supposons

$$
Z_{q}=\Sigma \frac{Z_{q}^{\sigma}}{\Gamma(\sigma+1) \Gamma(q-\sigma+1)} x^{2 n s+2 q-4 \sigma},
$$

où la sommation se rapporte à $\sigma$ et s'étend depuis $\sigma=0$ jusqu'à $\sigma=q$. Toute réduction faite, et ayant mis pour plus de simplicité $n^{2}-2 n s=\lambda, 2 n s=\mu$, on obtient pour $Z_{q}^{\sigma}$ l'expression

4.

$$
\begin{gathered}
{\left[-(q-\sigma) \lambda-\sigma \mu+(q-2 \sigma)^{2}\right] Z_{q}^{\sigma}} \\
+(q-\sigma)(\lambda-2 q+4 \sigma+2)(\lambda-2 q+4 \sigma+1) Z_{q-1}{ }^{\sigma} \\
+\sigma(\mu+2 q-4 \sigma+2)(\mu+2 q-4 \sigma+1) Z_{q-1}{ }^{\sigma-1} \\
+16 \sigma(q-\sigma)[\lambda \mu-2(q-2)(\lambda+\mu)] Z_{q-2}{ }^{\sigma-1}=0 .
\end{gathered}
$$

En supposant la valeur de $Z_{0}^{0}$ égale à l'unité, les valeurs de $Z_{q}{ }^{\sigma}$ se trouvent complétement déterminées; malheureusement la loi des coefficients n'est pas en évidence, excepté dans le cas de $\sigma=0$, ou $\sigma=q$. En calculant les termes successifs, on obtient

$$
\begin{aligned}
& z_{s}=(4 \alpha)^{s(n-2 s)} \cdot x^{2 n s} \\
& -(4 \alpha)^{s(n-2 s)-1}\left\{\begin{aligned}
& \frac{1}{1} \lambda \cdot x^{2 n s+2} \\
&+\frac{1}{1} \mu \cdot x^{2 n s-2}
\end{aligned}\right. \\
& +(4 \alpha)^{s(n-2 s)-2}\left\{\begin{array}{cc}
\frac{1}{1.2} & \lambda(\lambda-3) x^{2 n s+4} \\
+\frac{1}{1.1}\left(\lambda \mu+2-\frac{10 \lambda \mu}{\lambda+\mu}\right) x^{2 n s} \\
+\frac{1}{1.2} & \mu(\mu-3) x^{2 n s-4}
\end{array}\right. \\
& -(4 \alpha)^{s(n-2 s)-3}\left\{\begin{array}{lr}
\frac{1}{1.2 .3} & \lambda(\lambda-4)(\lambda-5) x^{2 n s+6} \\
+\frac{1}{1.2 .1} \lambda\left(\mu(\lambda-3)+40-\frac{20 \lambda \mu}{\lambda+\mu}\right) x^{2 n s+2} \\
+\frac{1}{1.1 .2} \mu\left(\lambda(\mu-3)+40-\frac{20 \lambda \mu}{\lambda+\mu}\right) x^{2 n s-2} \\
+\frac{1}{1.2 \cdot 3} \quad \mu(\mu-4)(\mu-5) x^{2 n s-6}
\end{array}\right.
\end{aligned}
$$

$+\& \mathrm{c}$. 
On aurait une valeur assez générale de $z$ en multipliant les différentes fonctions $z_{s}$ chacune par une constante arbitraire, et en sommant les produits; mais dans le cas actuel où $z$ dénote le dénominateur de $\sqrt{ } k \sin a m n u$, la valeur convenable de $z$ se réduit à

$$
z=z_{0} \pm z_{1} \ldots \pm z_{s} \ldots
$$

où $s$ est un nombre entier et positif, entre zéro et $\frac{1}{2} n$ ou $\frac{1}{2}(n-1)$. On aura par exemple dans le cas $n=5$ (les signes étant tous positifs si $n$ est impair, et alternativement positifs et négatifs si $n$ est pair), en supprimant les puissances négatives de a (lesquelles s'entredétruisent):

$$
\begin{aligned}
& z_{0}=1, \\
& z_{1}=64 \alpha^{3} x^{10}-\alpha^{2}\left(160 x^{8}+240 x^{12}\right)+\alpha\left(140 x^{6}+368 x^{10}+360 x^{14}\right) \\
& -\left(50 x^{4}+125 x^{8}+300 x^{12}+275 x^{16}\right), \\
& z_{2}=16 \alpha^{2} x^{20}-\alpha\left(80 x^{18}+20 x^{22}\right)+\left(170 x^{16}+62 x^{20}+5 x^{24}\right),
\end{aligned}
$$

et de là :

$$
\begin{aligned}
z=1 & -50 x^{4}+140 \alpha x^{6}-\left(125+160 \alpha^{2}\right) x^{8}+\left(368 \alpha+64 \alpha^{3}\right) x^{10}-\left(300+240 \alpha^{2}\right) x^{12} \\
& +360 \alpha x^{14}-105 x^{16}-80 \alpha x^{18}+\left(62+16 \alpha^{2}\right) x^{20}-20 \alpha x^{22}+5 x^{24}
\end{aligned}
$$

ce qui est effectivement la valeur de $z$ que j'ai trouvée dans le mémoire cité pour ce cas particulier. 